\title{
AVALIAÇÃO DA INTERAÇÃO DE VARIÁVEIS GEOMÉTRICAS SOBRE A EFICIÊNCIA DE SEPARAÇÃO, A RAZÃO DE LÍQUIDO E O DIÂMETRO DE CORTE REDUZIDO EM UM HIDROCICLONE OTIMIZADO
}

\author{
Y. N. KYRIAKIDIS ${ }^{1 *}$, F. F. SALVADOR ${ }^{1}$, M. A. S. BARROZO ${ }^{1}$, L. G. M. VIEIRA ${ }^{1}$ \\ ${ }^{1}$ Universidade Federal de Uberlândia, Faculdade de Engenharia Química \\ *e-mail: yannenk@yahoo.com.br
}

\begin{abstract}
RESUMO
Hidrociclones são separadores cônico-cilíndricos destinados à separação sólido-líquido ou líquido-líquido. Sua aplicação é amplamente difundida nos mais variados setores industriais e, a depender da finalidade almejada pelo usuário (concentração ou classificação), é possível potencializar o desempenho desses equipamentos mediante alterações de dimensões geométricas do hidrociclone. Assim, este trabalho teve como objetivo estudar experimentalmente o efeito da interação de duas variáveis geométricas (diâmetro do orifício de underflow e comprimento de vortex finder) sobre as respostas eficiência total, razão de líquido e diâmetro de corte reduzido em um hidrociclone que possui otimizadas as relações geométricas das principais variáveis de projeto. De acordo com os resultados experimentais, o hidrociclone em questão não apresentou alterações significativas em sua eficiência total na região de busca utilizada para ambas as variáveis analisadas, ao passo que em termos de razão de líquido, essa resposta foi influenciada significativamente apenas pelo diâmetro do orifício de underflow, apresentando comportamento linear e diretamente proporcional. Com relação ao diâmetro de corte reduzido, este também sofreu efeitos apenas do diâmetro do orifício de underflow, mas em menor intensidade do que a razão de líquido, apresentando comportamento linear e inversamente proporcional.
\end{abstract}

\section{INTRODUÇÃO}

Os hidrociclones são separadores cônico-cilíndricos geralmente destinados ao processamento de suspensões aquosas contendo uma fase particulada dispersa. Esses equipamentos são usualmente constituídos de uma parte cilíndrica acoplada a um tronco de cone, com uma entrada tangencial no topo da parte cilíndrica. Quando a suspensão é alimentada sob pressão no equipamento, a geometria do hidrociclone proporciona a geração de um campo centrífugo, que é responsável pela separação das fases.

Esses separadores possuem vasta aplicação industrial, uma vez que apresentam custo reduzido de aquisição e manutenção por não possuírem partes móveis. Assim, é possível encontrar hidrociclones nos mais diversos setores industriais, tais como o têxtil, o alimentício, o farmacêutico, o minerador, entre outros.

Os hidrociclones podem ser utilizados com o objetivo de clarificar uma suspensão (classificar partículas) ou de concentrar uma das correntes de saída do equipamento. A depender do propósito da separação, os hidrociclones assumem diferentes relações geométricas das suas principais dimensões características em razão do diâmetro da parte cilíndrica. Essas relações geométricas específicas caracterizam uma "família" de 
hidrociclones. Neste contexto, a Figura 1 apresenta genericamente as dimensões geométricas relevantes para o estudo do processo de hidrociclonagem.

Figura 1 - Principais dimensões geométricas de um hidrociclone

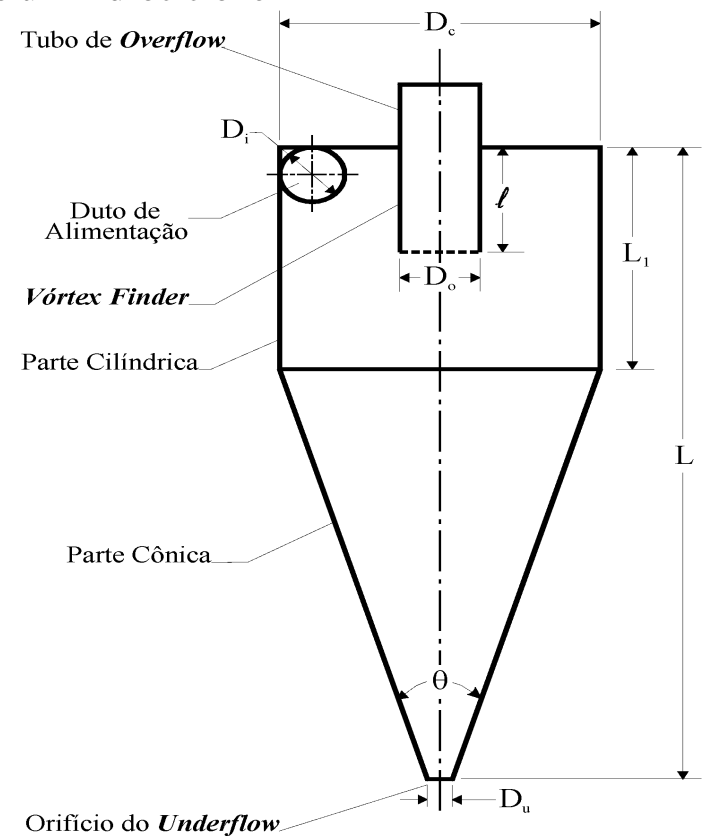

Fonte: Vieira (2006).

Considerando os resultados proporcionados pelas famílias tradicionais de hidrociclones e as reais necessidades do setor industrial, a Faculdade de Engenharia Química da Universidade Federal de Uberlândia (FEQUI/UFU) concentra seus esforços na otimização desses equipamentos, posto que a demanda desses separadores principalmente nas indústrias da região do Triângulo Mineiro e interior de Goiás se mostra elevada.

Neste contexto, Vieira (2006) estudou o desempenho de separação de hidrociclones de famílias não convencionais, oriundas de um Planejamento Composto Central (PCC), cujos fatores da matriz de planejamento incluíam as relações geométricas de famílias tradicionalmente conhecidas. Para tanto, foram analisadas as dimensões: diâmetro da alimentação (Di), diâmetro de overflow (Do), comprimento total (L) e ângulo do tronco de cone $(\theta)$. Do seu planejamento experimental, Vieira (2006) deu origem a 25 geometrias distintas de hidrociclones, as quais foram estudadas numérica e experimentalmente.

De acordo com as principais simulações fluidodinâmicas e dados experimentais obtidos por Vieira (2006), o autor concluiu que era possível conciliar, em um único equipamento, alta eficiência $(\eta=80,19 \%)$ com baixo gasto energético $(\mathrm{Eu}=1323)$. $\mathrm{O}$ hidrociclone do autor era extremamente classificador $\left(\mathrm{d}_{50}=5,04 \mu \mathrm{m}\right)$, porém $\mathrm{o}$ equipamento ainda descarregava uma quantidade significante do líquido da suspensão inicial na corrente de underflow $\left(\mathrm{R}_{\mathrm{L}}=36,56 \%\right)$.

Apesar dos bons resultados utilizando um Planejamento Composto Central, Vieira (2006) não valeu-se de técnicas de otimização global em seu trabalho, o que instigou Silva (2012) a continuar o processo de otimização em hidrociclones utilizando técnicas de Superfície de Resposta aliadas à técnica do Algoritmo de Evolução Diferencial.

Para isso, Silva (2012) desenvolveu funções objetivo para o Algoritmo de Evolução Diferencial tendo como base os resultados experimentais obtidos por Vieira (2006). Então, estudos de caso foram feitos a fim de minimizar o custo energético com uma restrição na resposta diâmetro de corte, e dessa forma, Silva (2012) obteve relações geométricas de projeto ótimas, diferentes daquelas encontradas nas famílias tradicionais e distintas das obtidas por Vieira (2006), caracterizando um hidrociclone inédito.

Por meio de ensaios experimentais, Silva (2012) observou que o novo hidrociclone proporcionou uma razoável eficiência de separação $(\eta=52,90 \%)$ a um baixo custo energético $(\mathrm{Eu}=788)$ para o material particulado empregado pelo autor (rocha fosfática). Apesar da resposta de eficiência total ter sido comprometida pela redução do gasto energético e o poder 
classificador do hidrociclone ser diminuído $\left(d_{50}=10,60 \mu \mathrm{m}\right)$, se comparado ao hidrociclone de Vieira (2006), o hidrociclone otimizado por Silva (2012) proporcionou uma maior concentração da corrente de underflow $\left(\mathrm{R}_{\mathrm{L}}=5,53 \%\right)$, ou seja, uma maior recuperação do líquido em suspensão.

Ainda que otimizadas as dimensões de projeto do hidrociclone para um estudo de caso específico, Silva (2012) manteve fixas as dimensões acessórias diâmetro do orifício de underflow $\left(\mathrm{D}_{\mathrm{U}}\right)$ e comprimento de vortex finder ( $)$. Assim, este trabalho teve como objetivo estudar experimentalmente o efeito da interação entre essas duas variáveis geométricas acessórias sobre as respostas eficiência total $(\eta)$, razão de líquido $\left(R_{L}\right)$ e diâmetro de corte reduzido (d' $\left.{ }_{50}\right)$ no hidrociclone otimizado por Silva (2012).

\section{MATERIAL E MÉTODOS}

Em seus experimentos, Silva (2012) encontrou intervalos ótimos de valores para as variáveis de projeto, buscando a otimização do gasto energético do equipamento. As relações geométricas entre as dimensões ótimas com o diâmetro da parte cilíndrica $\left(D_{C}\right)$ estão apresentadas na Tabela 1.

Tabela 1 - Intervalos ótimos das relações geométricas de projeto para redução do gasto energético.

\begin{tabular}{cc}
\hline Relação & Intervalo ótimo \\
\hline $\mathrm{D}_{\mathrm{i}} / \mathrm{D}_{\mathrm{c}}$ & 0,22 a 0,30 \\
$\mathrm{D}_{\mathrm{o}} / \mathrm{D}_{\mathrm{c}}$ & 0,30 a 0,40 \\
$\mathrm{~L}_{\mathrm{D}}$ & 6,46 a 8,74 \\
$\mathrm{~L}_{1} / \mathrm{D}_{\mathrm{c}}$ & 1,96 a 2,65 \\
$\theta$ & $8^{\circ}$ a $10^{\circ}$
\end{tabular}

Fonte: Silva (2012).

Neste trabalho, foram adotados os mesmos intervalos apresentados na Tabela 1 , sendo que o diâmetro da parte cilíndrica $\left(\mathrm{D}_{\mathrm{C}}\right)$ foi fixado em $30 \mathrm{~mm}$.

As partes constituintes dos hidrociclones foram construídas separadamente e acopladas segundo um sistema de travas mecânicas, de forma a possibilitar a troca de uma ou mais peças rapidamente.

Além das variáveis de projeto, foi preciso ainda especificar as dimensões acessórias do hidrociclone (diâmetro do orifício de underflow - $\mathrm{D}_{\mathrm{U}}$, comprimento de vortex finder $-\ell$ ). As dimensões ótimas dessas duas variáveis são definidas de acordo com o propósito do processo de hidrociclonagem, isto é, se o equipamento é utilizado para classificação ou concentração de partículas.

Assim, a fim de possibilitar o estudo sobre o consumo energético e a concentração da corrente de underflow, um Planejamento Fatorial a Três Níveis foi feito para os fatores diâmetro de orifício de underflow (DU) e comprimento de vortex finder ( () .

Neste trabalho, foram empregados intervalos entre 3,0 e 5,0 mm para $D_{U}$ e entre 1,0 e $12,0 \mathrm{~mm}$ para $\ell$. Considerando o procedimento experimental, as variáveis tiveram que ser codificadas para se adequarem ao planejamento. Dessa forma, a variável comprimento de vortex finder $(\ell)$ foi codificada em X1 e a variável diâmetro do orifício de underflow $\left(\mathrm{D}_{\mathrm{U}}\right)$ foi codificada em $\mathrm{X} 2$, conforme as Equações 1 e 2. O que resultou nas dimensões de $\mathrm{D}_{\mathrm{U}} \mathrm{e} \ell$ relatadas na Tabela 2 e na matriz de planejamento descrita na Tabela 3.

$\mathrm{X} 1=\frac{\ell[\mathrm{mm}]-6,5}{5,5}$

$\mathrm{X} 2=\frac{\mathrm{D}_{\mathrm{U}}[\mathrm{mm}]-4,0}{1,0}$ 
Tabela 2 - Valores das variáveis codificadas comprimento de vortex finder e diâmetro do orifício de underflow.

\begin{tabular}{ccc}
\hline $\mathrm{X} 1 / \mathrm{X} 2$ & $\ell(\mathrm{mm})$ & $\mathrm{D}_{\mathrm{U}}(\mathrm{mm})$ \\
\hline-1 & 1,0 & 3,0 \\
0 & 6,5 & 4,0 \\
+1 & 12,0 & 5,0 \\
\hline
\end{tabular}

Tabela 3 - Matriz do Planejamento Fatorial a Três Níveis e Dois Fatores.

\begin{tabular}{ccc}
\hline Hidrociclone & $\mathrm{X} 1$ & $\mathrm{X} 2$ \\
\hline 1 & -1 & -1 \\
2 & -1 & 0 \\
3 & -1 & +1 \\
4 & 0 & -1 \\
5 & 0 & 0 \\
6 & 0 & +1 \\
7 & +1 & -1 \\
8 & +1 & 0 \\
9 & +1 & +1 \\
\hline
\end{tabular}

A partir das dimensões mostradas na Tabela 2, foram fabricadas peças de vortex finder $(\ell)$ e de orifício de underflow (DU), cujas combinações do planejamento experimental originou nove configurações de hidrociclones diferentes a serem estudadas, como apresentado na Tabela 3.

Com relação ao material particulado, foram utilizadas suspensões de "quartzito" para a realização deste trabalho. Todo o material particulado empregado foi gentilmente cedido pela empresa ANEX Mineração, localizada em Itabirito (MG).

$\mathrm{O}$ material teve sua densidade real determinada através da técnica de picnometria por deslocamento de gás hélio no equipamento AccuPyc 1330 da Micromeritics ${ }^{\circledR}$, cujo valor encontrado foi igual a $2,74 \mathrm{~g} / \mathrm{cm}^{3}$.

Ao que tange o tamanho das partículas, o material foi analisado e sua distribuição granulométrica foi obtida utilizando o equipamento Mastersizer Microplus da
Malvern. A distribuição de tamanhos de partículas foi ajustada segundo o modelo RRB com parâmetros " $\mathrm{d}_{63,2}$ " e " $\mathrm{n}$ " iguais a $12,89 \mu \mathrm{m}$ e 0,94 , respectivamente. A equação resultante foi obtida com um coeficiente de correlação quadrático igual a 0,99 e tanto o modelo ajustado quanto os dados experimentais são mostrados na Figura 2.

Figura 2 - Distribuição granulométrica do material particulado.

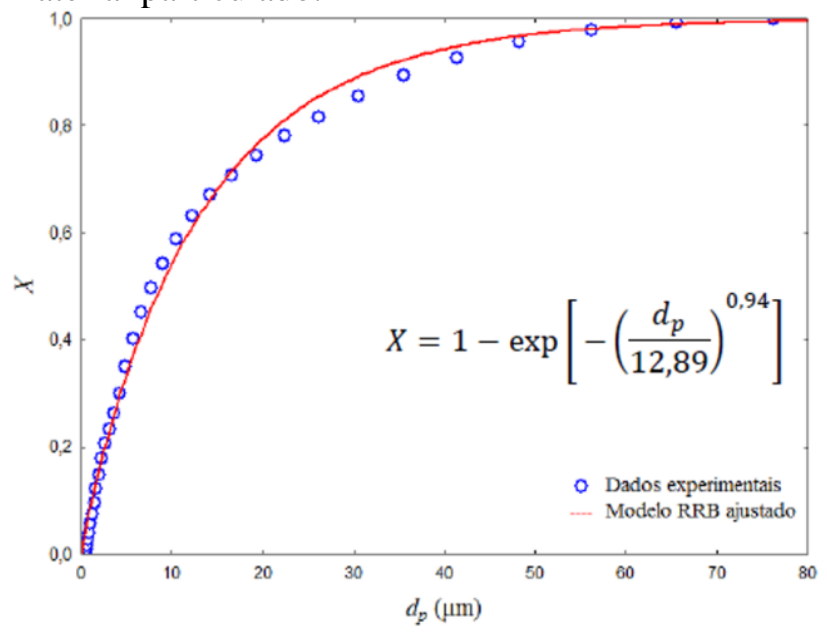

Já no que concerne à unidade experimental, esta era constituída de um tanque para armazenamento da suspensão de concentrado de "quartzito", bem como de chicanas e um agitador mecânico acoplado a um motor cuja função era homogeneizar a suspensão. As suspensões utilizadas eram mantidas na concentração de aproximadamente $1 \%$ (volume). Uma bomba do tipo helicoidal foi utilizada para alimentar a suspensão no hidrociclone, ao passo que para aferir a queda de pressão, foi utilizado um manômetro de Bourdon digital, instalado na linha de alimentação do hidrociclone. Foram utilizadas as seguintes condições de queda de pressão: 88, 118, 147 e $177 \mathrm{kPa}$.

No procedimento experimental empregado, para cada queda de pressão, as vazões volumétricas de alimentação e as concentrações mássicas das correntes foram obtidas através de técnicas gravimétricas, sendo que as concentrações foram aferidas a 
partir de amostras devidamente coletadas, pesadas em balança semi-analítica e secas em estufa por $24 \mathrm{~h}$. Além das amostras citadas, foram coletadas alíquotas da suspensão de alimentação e da corrente de underflow a fim de estimar a distribuição granulométrica das duas correntes.

Então, as respostas eficiência total ( $\eta)$, razão de líquido $\left(\mathrm{R}_{\mathrm{L}}\right)$ e diâmetro de corte reduzido $\left(\mathrm{d}_{50}\right)$ puderam ser obtidas por meio de procedimentos clássicos da literatura (MASSARANI, 1989 e SVAROVSKY, 1984), como mostram as Equações 3 a 5.

$\eta=\frac{\mathrm{C}_{\mathrm{WU}} \mathrm{W}_{\mathrm{U}}}{\mathrm{C}_{\mathrm{WA}} \mathrm{W}}$

$R_{L}=\frac{Q_{U}\left(1-C_{V U}\right)}{Q\left(1-C_{V_{A}}\right)}$

$0,5=$

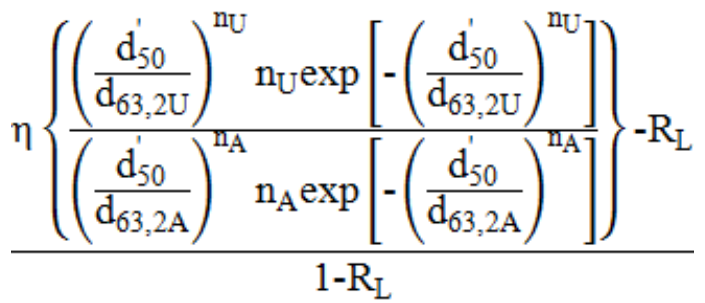

Além dos cálculos de $\eta, R_{L}$ e d' 50 , regressões múltiplas foram realizadas $(\alpha=5 \%)$ a fim de se estimar equações de Superfície de Resposta para cada uma das grandezas em questão.

\section{RESULTADOS E DISCUSSÃO}

Após a realização dos experimentos, as respostas analisadas neste estudo (eficiência total - $\eta$, razão de líquido $-\mathrm{R}_{\mathrm{L}}$, diâmetro de corte reduzido - d' ${ }_{50}$ ) foram calculadas para cada queda de pressão. Em seguida, foi feita a média dos resultados para cada equipamento do Planejamento Fatorial, conforme apresentado na Tabela 4.
Tabela 4 - Resultados experimentais obtidos.

\begin{tabular}{cccc}
\hline Hidrociclone & $\eta(\%)$ & $\mathrm{R}_{\mathrm{L}}(\%)$ & $\mathrm{d}_{50}^{\prime}(\mu \mathrm{m})$ \\
\hline 1 & 66,25 & 0,99 & 13,56 \\
& $( \pm 0,93)$ & $( \pm 0,03)$ & $( \pm 0,74)$ \\
& & & \\
2 & 69,50 & 5,08 & 11,79 \\
& $( \pm 1,63)$ & $( \pm 0,61)$ & $( \pm 0,89)$ \\
3 & 71,68 & 7,42 & 11,10 \\
& $( \pm 1,10)$ & $( \pm 0,67)$ & $( \pm 0,90)$ \\
4 & 70,35 & 0,97 & 13,75 \\
& $( \pm 0,96)$ & $( \pm 0,02)$ & $( \pm 0,82)$ \\
& 75,82 & 2,73 & 11,22 \\
5 & $( \pm 1,52)$ & $( \pm 0,44)$ & $( \pm 0,92)$ \\
& 72,64 & 6,00 & 11,21 \\
6 & $( \pm 1,41)$ & $( \pm 0,73)$ & $( \pm 0,92)$ \\
& & & \\
& 70,83 & 0,90 & 13,11 \\
7 & $( \pm 0,46)$ & $( \pm 0,01)$ & $( \pm 1,06)$ \\
& & & \\
& 70,53 & 2,74 & 12,13 \\
8 & $( \pm 1,07)$ & $( \pm 0,46)$ & $( \pm 0,77)$ \\
& & & \\
& 82,17 & 6,19 & 10,28 \\
& $( \pm 1,57)$ & $( \pm 0,58)$ & $( \pm 0,70)$ \\
\hline
\end{tabular}

Através de regressões múltiplas realizadas, observou-se que a eficiência total dos equipamentos permaneceu praticamente inalterada em todos os casos, uma vez que não foi possível estimar uma equação que correlacionasse estatisticamente essa resposta com os fatores X1 e X2.

Por outro lado, os resultados de razão de líquido $\left(\mathrm{R}_{\mathrm{L}}\right)$ possibilitaram o ajuste de uma Superfície de Resposta, como apresentado na Equação $6\left(R^{2}=0,90\right)$.

$$
\begin{aligned}
\mathrm{R}_{\mathrm{L}}=3,67+\left[\begin{array}{ll}
0 & 2,79
\end{array}\right]\left[\begin{array}{l}
\mathrm{X} 1 \\
\mathrm{X} 2
\end{array}\right]+ \\
+\left[\begin{array}{ll}
\mathrm{X} 1 & \mathrm{X} 2
\end{array}\right]\left[\begin{array}{ll}
0 & 0 \\
0 & 0
\end{array}\right]\left[\begin{array}{l}
\mathrm{X} 1 \\
\mathrm{X} 2
\end{array}\right]
\end{aligned}
$$

A Equação 6 indica que a resposta $R_{L}$ não sofreu alterações devido às mudanças 
impostas em X1. Porém, a mesma equação sugere que a razão de líquido depende linear e diretamente do fator $\mathrm{X} 2$, ou seja, da variável diâmetro do orifício de underflow $\left(\mathrm{D}_{\mathrm{U}}\right)$, como mostra a Figura 3.

Figura 3 - Superfície de Resposta estimada para a razão de líquido $\left(\mathrm{R}_{\mathrm{L}}\right)$.

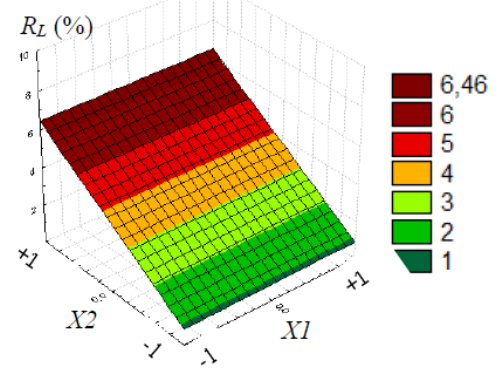

Os resultados apontaram um aumento considerável na recuperação de líquido para menores valores de $\mathrm{X} 2$, visto que a razão de líquido $\left(\mathrm{R}_{\mathrm{L}}\right)$ diminuiu de aproximadamente $6,46 \%$ para $1,00 \%$, se em vez de utilizado o $\mathrm{D}_{\mathrm{U}}$ de $5 \mathrm{~mm}(\mathrm{X} 2=+1)$ fosse empregado o $\mathrm{D}_{\mathrm{U}}$ de $3 \mathrm{~mm}(\mathrm{X} 2=-1)$. Tal acontecimento refletiu na ordem de grandeza obtida para o efeito linear do fator X2 (2,79), que foi similar à ordem de grandeza da média $(3,67)$, como mostra a Equação 6.

Analisando os resultados de diâmetro de corte reduzido (d'50), também foi possível estimar uma Superfície de Resposta para essa variável, como mostra a Equação 7 e Figura 4 $\left(\mathrm{R}^{2}=0,87\right)$.

$$
\begin{aligned}
& \mathrm{d}_{50}^{\prime}=12,02+\left[\begin{array}{ll}
0 & -1,31
\end{array}\right]\left[\begin{array}{l}
\mathrm{X} 1 \\
\mathrm{X} 2
\end{array}\right] \\
&+\left[\begin{array}{ll}
\mathrm{X} 1 & \mathrm{X} 2
\end{array}\right]\left[\begin{array}{ll}
0 & 0 \\
0 & 0
\end{array}\right]\left[\begin{array}{l}
\mathrm{X} 1 \\
\mathrm{X} 2
\end{array}\right]
\end{aligned}
$$

A Equação 7 sugere que a resposta analisada $\left(\mathrm{d}_{50}{ }_{50}\right)$ depende exclusivamente do fator X2 e, ainda, que a relação entre essas duas grandezas é linear e inversamente proporcional. $\mathrm{Ou}$ seja, quanto maior o diâmetro do orifício de underflow $\left(\mathrm{D}_{\mathrm{U}}\right)$, menor o diâmetro de corte reduzido $\left(\mathrm{d}_{50}\right)$ e maior a classificação de partículas, uma vez que partículas de menores diâmetros foram coletadas na corrente de underflow.

Figura 4 - Superfície de Resposta estimada para o diâmetro de corte reduzido (d' $\left.{ }_{50}\right)$.

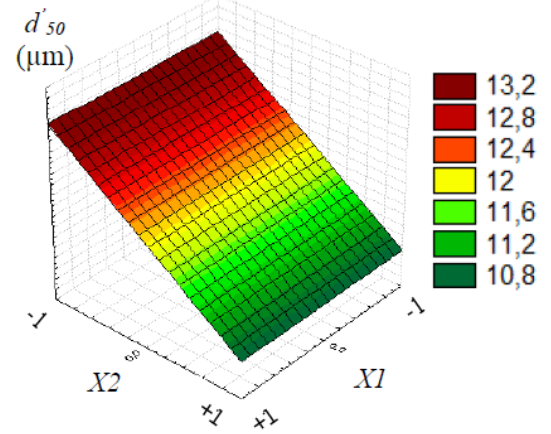

Entretanto, de acordo com a Equação 7, o efeito linear encontrado para o fator X2 $(-1,31)$ foi quase 12 vezes inferior à média $(12,02)$. Portanto, pode-se inferir que alterações em X2 tem um impacto menor sobre d' 50 do que sobre $\mathrm{R}_{\mathrm{L}}$.

\section{CONCLUSÃO}

Com base nos resultados experimentais, os hidrociclones estudados através do Planejamento de Experimentos não apresentaram diferenças significativas na resposta eficiência total $(\eta)$, ao passo que para a razão de líquido $\left(\mathrm{R}_{\mathrm{L}}\right)$ e o diâmetro de corte reduzido $\left(\mathrm{d}_{50}\right)$ apenas a variável geométrica $\mathrm{D}_{\mathrm{U}}$, representada pelo fator $\mathrm{X} 2$, influenciou diretamente os resultados obtidos.

Pelas Superfícies de Resposta ajustadas, foi possível notar que uma diminuição do diâmetro do orifício de underflow $\left(\mathrm{D}_{\mathrm{U}}\right)$ proporcionou menores valores de $\mathrm{R}_{\mathrm{L}}$. Portanto uma maior quantidade de líquido foi recuperada. Mas o aumento em $D_{U}$ também fez com que d' 50 fosse majorado, e partículas maiores deixassem de ser coletadas.

Todavia, foi observado que alterações em $D_{U}$ provocaram um efeito de maior intensidade sobre $R_{L}$ do que sobre a resposta de d' 50 . 
Portanto, considerando as três respostas analisadas e a região de busca delimitada no Planejamento de Experimentos, concluiu-se que o diâmetro de $3 \mathrm{~mm}$ para o orifício de underflow $\left(\mathrm{D}_{\mathrm{U}}\right)$ mantém o alto patamar de eficiência total e majora o poder concentrador do hidrociclone sem grandes prejuízos à classificação das partículas. Já o comprimento de vortex finder ( () não teve efeitos relevantes sobre as respostas analisadas neste trabalho, visando a melhoria de separação no hidrociclone estudado.

\section{NOMENCLATURA}

$\mathrm{C}_{\mathrm{VA}}$ - concentração volumétrica de sólidos na corrente de alimentação [-]

$\mathrm{C}_{\mathrm{VU}}$ - concentração volumétrica de sólidos na corrente de underflow [-]

$\mathrm{C}_{\mathrm{WA}}$ - concentração mássica de sólidos na corrente de alimentação [-]

$\mathrm{C}_{\mathrm{WU}}$ - concentração mássica de sólidos na corrente de underflow [-]

$\mathrm{d}_{50}$ - diâmetro de corte reduzido [L]

$\mathrm{d}_{63,2}$ - parâmetro do modelo RRB [L]

$\mathrm{d}_{63,2 \mathrm{~A}}$ - parâmetro do modelo RRB na corrente de alimentação [L]

$\mathrm{d}_{63,2 \mathrm{U}}$ - parâmetro do modelo RRB na corrente de underflow [L]

$d_{P}-$ diâmetro volumétrico da partícula [L]

$\mathrm{D}_{\mathrm{C}}$ - diâmetro da seção cilíndrica [L]

$\mathrm{D}_{\mathrm{i}}$ - diâmetro do duto de alimentação [L]

$\mathrm{D}_{\mathrm{O}}$ - diâmetro do orifício de overflow [L]

$\mathrm{D}_{\mathrm{U}}$ - diâmetro do orifício de underflow [L]

$\mathrm{Eu}$ - número de Euler [-]

$\ell$ - comprimento de vortex finder [L]

$\mathrm{L}$ - comprimento total do hidrociclone [L]

$\mathrm{L}_{1}$ - comprimento da seção cilíndrica [L]

$\mathrm{n}$ - parâmetro do modelo RRB [-]

$\mathrm{n}_{\mathrm{A}}$ - parâmetro do modelo RRB na corrente de alimentação [-]

$\mathrm{n}_{\mathrm{U}}$ - parâmetro do modelo RRB na corrente de underflow [-]

Q - vazão volumétrica de suspensão na corrente de alimentação $\left[\mathrm{L}^{3} \mathrm{~T}^{-1}\right]$
Qu - vazão volumétrica de suspensão na corrente de underflow $\left[\mathrm{L}^{3} \mathrm{~T}^{-1}\right]$

$\mathrm{R}^{2}$ - coeficiente de correlação quadrático [-]

$\mathrm{R}_{\mathrm{L}}$ - razão de líquido [-]

$X$ - fração mássica de partículas na corrente de alimentação com diâmetro igual ou inferior a $d_{P}[-]$

X1 - variável codificada 1 representativa de $\ell$ $[-]$

X2 - variável codificada 2 representativa de $\mathrm{D}_{\mathrm{U}}[-]$

W - vazão mássica da corrente de alimentação $\left[\mathrm{M}^{1} \mathrm{~T}^{-1}\right]$

$\mathrm{W}_{\mathrm{U}}$ - vazão mássica da corrente de underflow $\left[\mathrm{M}^{1} \mathrm{~T}^{-1}\right]$

$\alpha$ - nível de significância [-]

$\eta$ - eficiência total de separação [-]

$\theta$ - ângulo de abertura da parte cônica $\left[{ }^{\circ}\right]$

\section{REFERÊNCIAS}

MASSARANI, G. Projeto e análise do desempenho de ciclones e hidrociclones ii. In: XVII Encontro Nacional sobre Escoamento em Meios Porosos, 1989, São Carlos. Anais... São Carlos, 1989, p. 125-136.

SILVA, D. O. Hidrociclones: Um Estudo de Otimização e Análise do Efeito das Variáveis Geométricas Utilizando Técnicas Experimentais e de CFD. 2012. 189f. Tese (Doutorado em Engenharia Química) Universidade Federal de Uberlândia, Uberlândia, 2012.

SVAROVSKY, L. Hydrocyclones. Eastbourne, UK: Holt, Rinehart \& Winston, 1984.

VIEIRA, L. G. M. Otimização dos Processos de Separação em Hidrociclones Filtrantes. 2006. 298f. Tese (Doutorado em Engenharia Química) - Universidade Federal de Uberlândia, Uberlândia, 2006. 


\section{AGRADECIMENTOS}

Os autores agradecem à CAPES, à FAPEMIG e ao CNPq pelo apoio financeiro, bem como à Universidade Federal de Uberlândia (UFU), à Faculdade de Engenharia Química (FEQUI/UFU) e ao Laboratório de Separação e Energias Renováveis (LASER-FEQUI/UFU). 